\title{
Catheter detection and segmentation in volumetric ultrasound using SVM and GLCM
}

\author{
V.V. Danilov ${ }^{1}$, I.P. Skirnevskiy², R.A. Manakov³, D. Yu. Kolpashchikov4, O.M. Gerget5, F. Melgani6* \\ Medical Devices Design Laboratory, Tomsk Polytechnic University, Tomsk, Russia \\ * Signal Processing and Recognition Laboratory, University of Trento, Trento, Italy

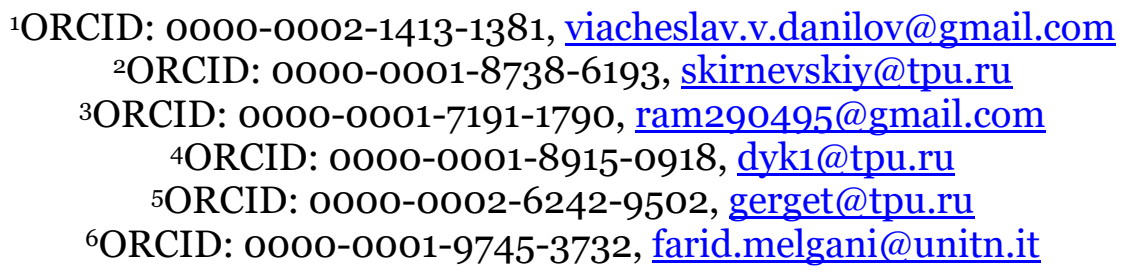

\begin{abstract}
The focus of this study was to develop an image-based algorithm for the catheter detection and segmentation in volumetric ultrasound. Nowadays, echocardiography is one of the most common methods of cardiovascular diseases diagnostic and surgery. As an input data the algorithm uses epicardial full-volume $3 \mathrm{D}$ echocardiography datasets. In total, 9 datasets consisted of 15 three-dimensional timeframes were processed. Each 3D timeframe includes 208 slices with the size of $176^{*} 176$. To correctly detect the catheter, the feature-based approach was applied to recognition the catheter within the $3 \mathrm{D}$ echocardiography datasets. MATLAB was used for all calculations as the main numerical computing environment. Before the main part of the algorithm, we performed preprocessing of the data. The pre-processing workflow comprises imposing a restriction on the area of the region for noise reduction, automatic Otsu's thresholding and morphological operations.

The proposed algorithm based on gray-level co-occurrence matrix (GLCM) was applied as a feature extraction technique. Once the GLCM was computed, we obtained correlation, contrast, homogeneity and energy features. Then we applied feature thresholds to the catheter detection. These thresholds were obtained using Support Vector Machine (SVM) with the linear kernel function and standardization the predictor data. The average segmentation and recognition accuracies of the algorithm equal $94.16 \%$ and $87.2 \%$ respectively. The processing time for one $2 \mathrm{D}$ slice and one $3 \mathrm{D}$ dataset are equal to $9 \pm 0.2$ milliseconds and $1.96 \pm 0.045$ seconds, respectively. Though the algorithm is not timeconsuming for $2 \mathrm{D}$ mode, it is still complicated to apply it to $3 \mathrm{D}$ real-time visualization.
\end{abstract}

Keywords: catheter detection, catheter segmentation, texture analysis, GLCM, SVM, ultrasound.

\section{Introduction}

Image processing and visualization tools are an indispensable part during performing microsurgical endovascular procedures with catheters on the heart. Existing visualization and image processing methods limit the range of complex manipulations performed on the beating heart. In its turn, classical visualization techniques (echocardiography, fluoroscopy, and angiography) [1-3] possess several crucial disadvantages such as insufficient quality of the output data, high level of noise and limited scope (field of view). These drawbacks significantly reduce the range of possible transcatheter procedures on the heart by means of endovascular technology and also hinder the development of this area. Two-dimensional echocardiography (EchoCG) does not allow adequately displaying the spatial position of the medical instrument (catheter) and visualize the area of interest. Additionally, 
3D EchoCG displays a limited area with a significant level of digital noise, shadows, and artifacts. Thus, for today, a wide range of cardiosurgical operations are performed only on an open heart using artificial circulation $[4,5]$. Such methods of pathology correction have a number of shortcomings associated with severe postoperative complications and long-term rehabilitation, which is an important problem in cardiology. The availability of new methods of visualization, image processing and delivering catheter devices allows performing a number of interventions on the working heart, including stenting of vessels, radiofrequency ablation in cases of cardiac arrhythmias, closing of atrial and interventricular septal defects. Currently, there are a few numbers of research studies that are devoted to the application of recognition and tracking of medical devices.

Nowadays biplane fluoroscopy that uses $\mathrm{X}$-ray image intensifier is one of the most frequently used methods of visualization (up to 60\%) [6]. In the studies of Matthias Hoffman, a semi-automatic reconstruction of the catheter is performed based on two projections $[7,8]$. The authors minimized the interaction with the user in their algorithm; nevertheless, recognition still requires the assigning of at least one point belonging to the catheter area. The accuracy of the methods is at a high level, but the total processing time takes about 8 seconds, which confirms the impossibility of using this method in real time. Moreover, the Xray based methods are not applicable for heart imaging, because they cannot provide soft tissue information.

Research study on the skeletonization and visualization of the catheter in the threedimensional space of the vasculature were carried out by Baert Shirley, Van de Kraats Everine and others [9]. Another research devoted to the detection and tracking of different types of catheters was performed by Ying Liang Ma et al. [10]. In their study, scientists did not reconstruct the catheter but only used the tracking algorithm for three different types of catheters.

In the other work, P. Ambrosini et al. proposed a method, based on a hidden
Markov model, for $3 \mathrm{D}$ catheter tip tracking with $2 \mathrm{D}$ X-ray catheterization sequence and $3^{\mathrm{D}}$ rotational angiography [11]. But in the research intraoperative images are enhanced using contrast agent for visualizing the vasculature.

In paper [12] the authors describe a method based on registration X-rays and echocardiography. The presented algorithm had an average error of less than $1 \mathrm{~mm}$ and the speed could reach 1.5 fps. However, the combination of $\mathrm{X}$-rays and ultrasound signals impose restrictions on the structure of input data end equipment. In this regard, the main scientific direction of this study is the research and development of the algorithm for intraoperative imaging and catheter visualization using the $3 \mathrm{D}$ echocardiography data.

\section{Materials}

\subsection{Data description}

Epicardial full-volume

echocardiography datasets were acquired on the porcine heart using an $\mathrm{X}_{7-2 \mathrm{t}}$ transducer on a Philips iE33 machine and PMS5.1 Ultrasound software (Philips Healthcare, Andover MA). During the data acquisition step, 9 datasets were acquired. Each dataset consists of 15-17 timeframes and each of them includes 208 slices of $176^{*} 176$-pixel size. Example of the data acquiring using Philips iE33 echocardiographer is shown in Fig. 1.

The experimental protocols were approved by the Boston Children's Hospital Institutional Animal Care and Use Committee (IACUC).

The datasets were processed offline on the computer equipped with Intel Core i74790K 4.0 GHz CPU and NVIDIA GeForce 960 GT using MATLAB (MathWorks, Natick MA). 


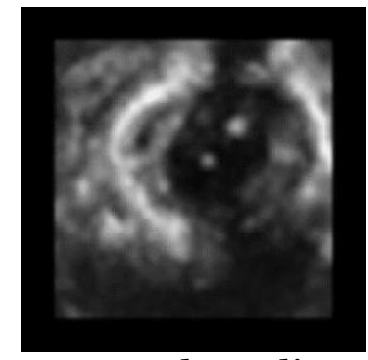

Fig. 1. An exemplary slice of the data.

\subsection{Methods}

\subsubsection{Workflow}

To efficiently recognize and segment the catheter we used sequential steps with particular options and setting for each of them. Features selected for the algorithm are the most relevant properties that can be used for the catheter recognition. The main steps of the algorithm are presented as cascadebased workflow shown in Fig. 2.

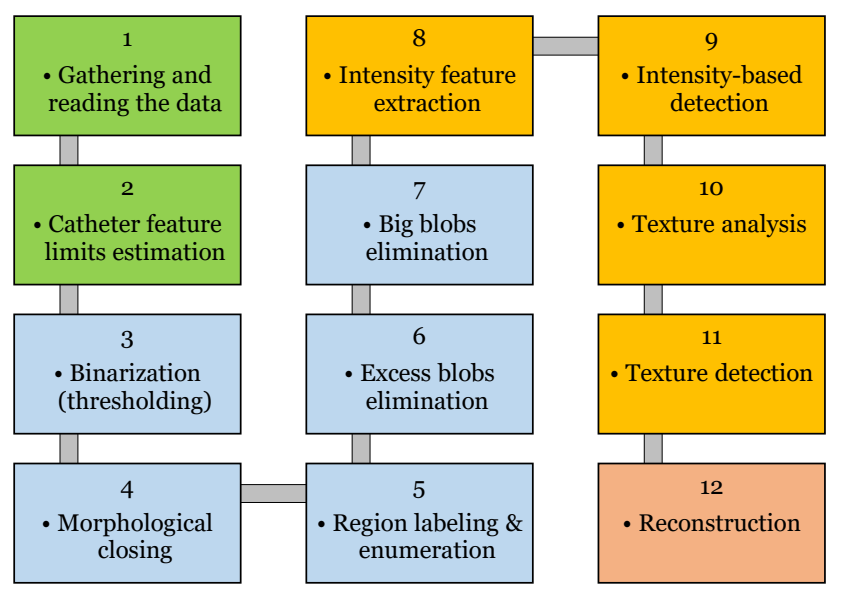

Fig. 2. Basic steps for recognition and segmentation algorithm of the catheter: green blocks - data acquisition and feature studying, blue blocks - coarse detection and segmentation, orange blocks - delicate detection and segmentation, red block smoothed $3 \mathrm{D}$ reconstruction.

To gather the whole data, we used realtime streaming of Philips iE33 machine. After getting all the medical data, we transfer it from DICOM Philips format into Nearly Raw Raster Data (NRRD) format.

\subsubsection{Morphology}

In regard to the initial processing methods, we applied automatic Otsu's thresholding for binarization an image [13] and morphological closing based on morphological reconstruction for filling the holes [14]. The disk-shaped element was used as the main structuring element for this morphological procedure. Using a disk structuring element allows preserving the circular nature of the object. In our case, the catheter has the circular or elliptical shape depending on its position and the position of the ultrasound transducer.

Small artifacts and noise emissions were removed by the imposing area restrictions. The output of the previous steps was a set of labeled regions including the region of interest (catheter). However, among these regions, there are false ones (see Fig. 4b).

\subsubsection{Feature selection}

We put forward a hypothesis that, by imposing different feature constraints, the catheter can be accurately found in the image. We selected several high-impact features such as ROI area, mean and standard deviation of intensity within a region and four textural features such as contrast, correlation, energy, and homogeneity. In order to limit the number of obtained regions, we calculated values of all features and entered limits for them, which are equal to ranges, corresponding to the region of the catheter. Textural features were calculated using gray-level co-occurrence matrix (GLCM) [15,16]. The GLCM for an exemplary slice is shown in Fig. 3.

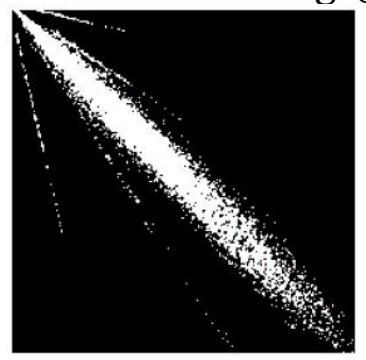

Fig. 3. GLCM for an exemplary slice.

\subsubsection{GLCM}

In order to compute textural features, GLCM should be normalized, so that the sum of its elements is equal to 1. Each element $(r, c)$ in the normalized GLCM is the joint probability occurrence of pixel pairs with a defined spatial relationship having gray level values $r$ and $c$ in the image.

Statistical properties of the image derived from GLCM are following: 
Contrast is a measure of the intensity variance or inertia between a pixel and its neighbor over the whole image. Contrast range for GLCMs $\left(255^{*} 255\right.$ size $)$ is from o to 64516. For the constant image, contrast is equal to 0 . Contrast is calculated as follows:

$$
\text { Contrast }=\sum_{i, j=0}^{N-1}|i-j|^{2} p(i, j)
$$

1. Correlation is a measure of how correlated a pixel is to its neighbor over the whole image. Correlation varies from 1 to -1 . Perfectly positively or negatively correlated image corresponds to 1 or -1 for correlation. Correlation is calculated as follows:

$$
\text { Correlation }=\sum_{i, j=0}^{N-1} \frac{(i-\mu i)(j-\mu j) p(i, j)}{\sigma_{i} \sigma_{j}}
$$

2. Energy is the sum of squared elements in the GLCM and it ranges from $o$ to 1. Energy is calculated as follows:

$$
\text { Energy }=\sum_{i, j=0}^{N-1} p(i, j)^{2}
$$

3. Homogeneity is a value that measures the closeness of the distribution of elements in the GLCM to the GLCM diagonal.

where:

$$
\text { Homogeneity }=\sum_{i, j=0}^{N-1} \frac{p(i, j)}{1+|i-j|}
$$

$p(i, j)$ is an element $(i, j)$ of the normalized symmetrical GLCM;

$N$ is the number of gray levels;

$\mu$ is the GLCM mean (being an estimate of the intensity of all pixels in the relationships that contributed to the GLCM), calculated as follows:

$$
\mu=\sum_{i, j=0}^{N-1} i p(i, j)
$$

$\sigma$ is the variance of intensities of all reference pixels in the relationships that contributed to the GLCM, calculated as:

$$
\sigma=\sum_{i, j=0}^{N-1} p(i, j)(i-\mu)^{2}
$$

Most regions similar to the region of the catheter were excluded after application delicate detection based on texture features and Gray-Level Co-Occurrence Matrix. It should also be noted, that threshold levels for textural and intensity features were empirically found.

The GLCM is a widely used technique, which is applied not only to the feature extraction but also for segmentation tasks. For instance, A. Rampun, H. Strange et al. proposed a segmentation method based on consideration each feature at two different configurations in the paper [17]. Mahesh B. Nagarajan, Markus B. Huber et al. used the GLCM for the lesion segmentation in the work [18].

\subsubsection{Image processing}

After acquiring the data and figuring out the catheter feature distribution, each slice is processed by the automatic thresholding algorithm. The result of the application of Otsu's thresholding method is shown in Fig. 4a.

The morphological closing follows the thresholding step and performs a flood-fill operation on background pixels (see Fig. 4b). This operation fills the holes and determines the connected area. At this step, we used 4connected neighborhoods connectivity. As it can be seen in Fig. 4b, the number of objects of these steps is 11, where the catheter is represented by the region 6 .

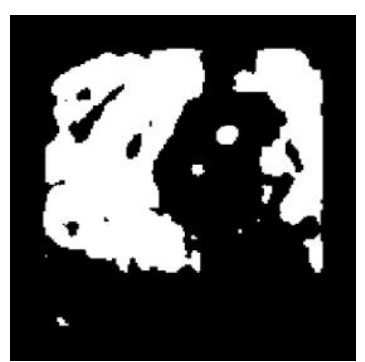

a)

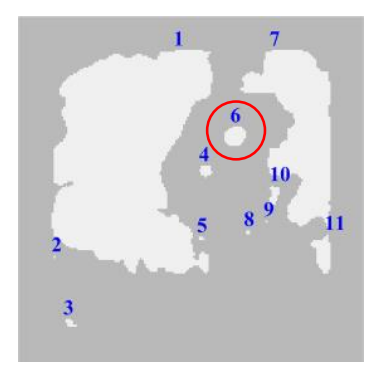

b)
Fig. 4. Automatic thresholding (a) and floodfill segmentation (b).

As we described above, one of the workflow steps is an intermediate procedure connected with excess blobs elimination. This step assumes that the region of the catheter cannot be smaller than 5 pixels even for an edged slice of the distal end of the catheter. This is why regions 2 and 9 reflected in Fig. 4b and shown in Fig. 5a in 
red circles were removed. The output of this step is a black and white (BW) mask with 9 objects.

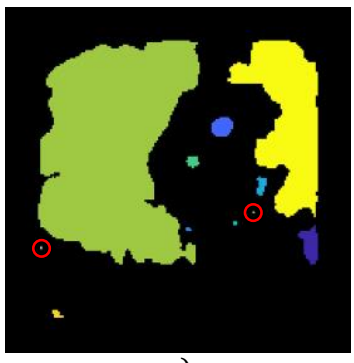

a)

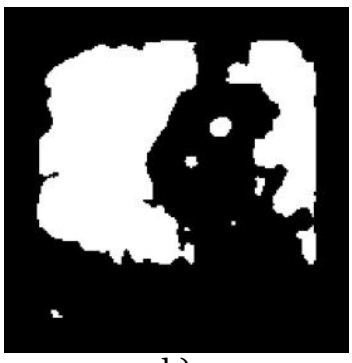

b)
Fig. 5. Excess blobs elimination: colorized input (a) and BW output (b).

As illustrated in Fig. 6 most regions are removed after step 7 of the workflow but there are 4 excess regions that meet the area criteria as well as the region of the catheter.

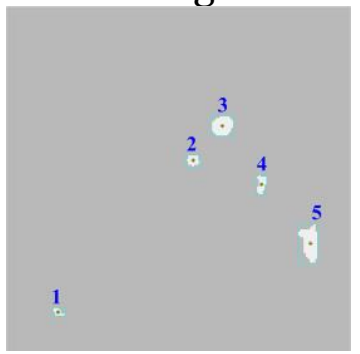

Fig. 6. Exclusion of the regions by area criteria.

After finishing the coarse detection and segmentation, we apply intensity analysis with further segmentation using the features. The first two features related to this step are the intensity mean and standard deviation of a certain region. To detect the region of the catheter, the mean and standard deviation were computed for each region on each slice of the dataset. The distribution of these two features for the current slice is presented in Fig. 7, where the average intensity and standard deviation for the catheter are 112 and 27, respectively. In its turn, several regions significantly differ from the region of the catheter, for example, region 1 and 4. In this regard, the obvious difference between the two classes (tissue and catheter) allowed linear SVM to set a threshold value for the features.

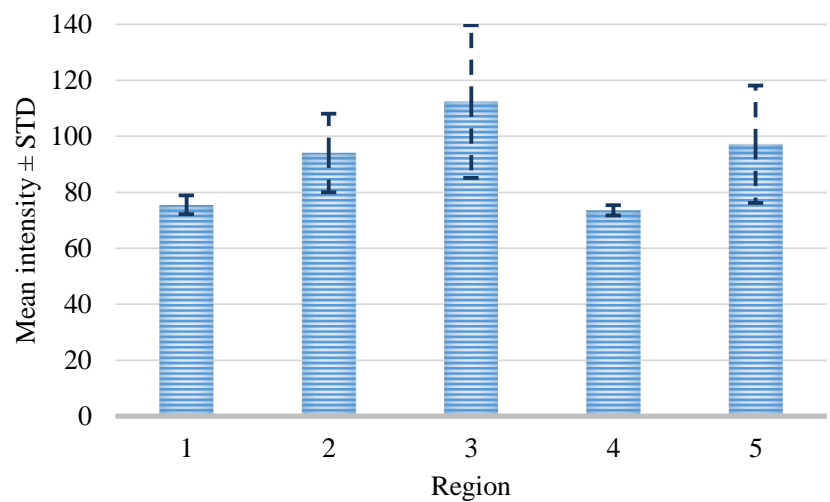

Fig. 7. Intensity analysis for the regions.

Values of these features for each region are compared to limits and are defined whether they meet the criteria or not. Fig. 8 displays how these two features excluded one of the redundant regions.

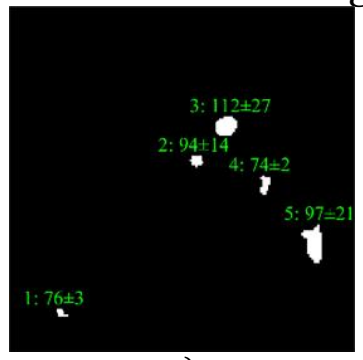

a)

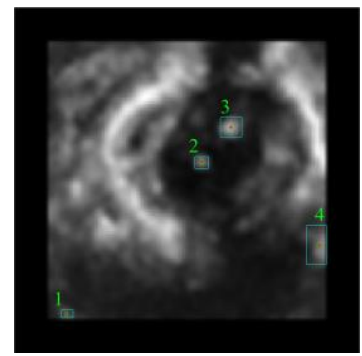

b)
Fig. 8. Intensity analysis (a) with the following intensity-based detection using mean and standard deviation (b).

The most precise step of the workflow is texture analysis with the GLCM. During this stage, four parameters such as the contrast, homogeneity, correlation, and energy are computed using GLCMs of each region.

Four GLCMs for remained regions are shown in Fig. 9. Afterward, textural features were compared with established limits. Computed feature values of the texture analysis shown below in Fig. 10 were compared to the reference ones shown in Fig. 12. 


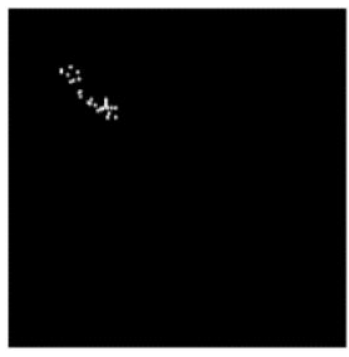

Region 1

a)

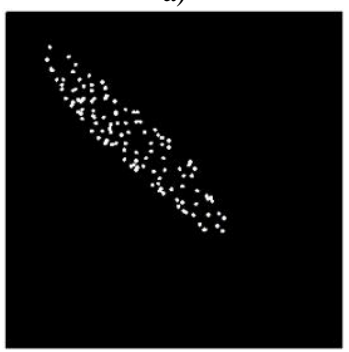

Region 3 (catheter)

c)

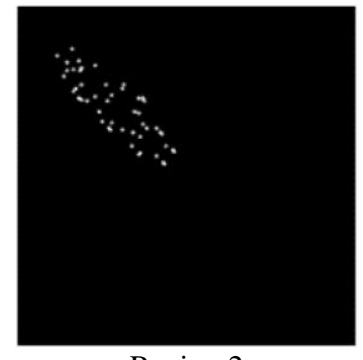

Region 2

b)

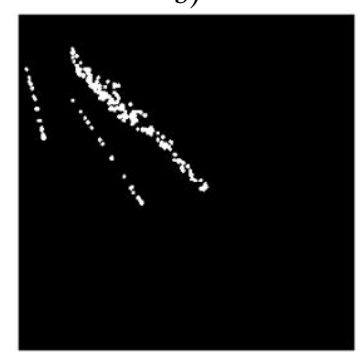

Region 4 d)

Fig. 9. Visual representation of GLCMs for remained regions.

The catheter (region 3) and two other regions (region 1 and 2) in Fig. 10 have sufficient uniformity, as indicated by the arrangement of the matrix elements on the central diagonal. In turn, the region 4 has internal brightness differences. Since the GLCM uniformity of the region cannot be used as a feature of the catheter. The correlation, contrast, homogeneity, and energy were computed based on the obtained data. Further processing was performed on the obtained features.

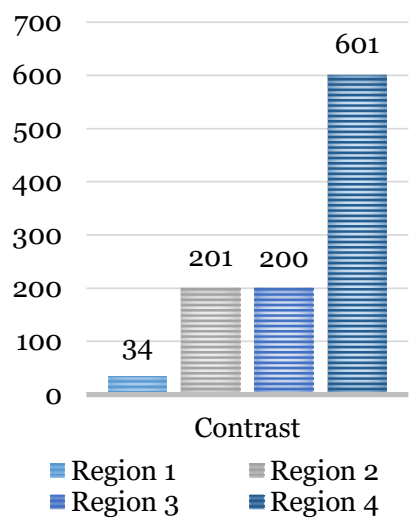

a)

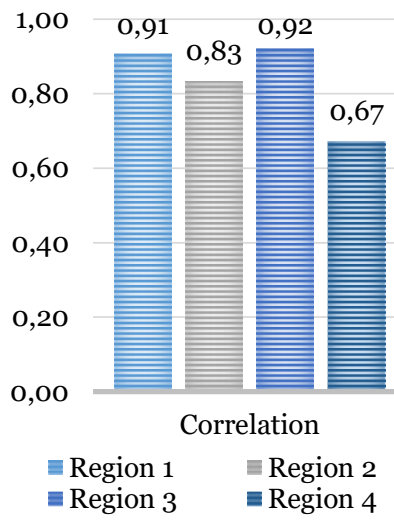

b)
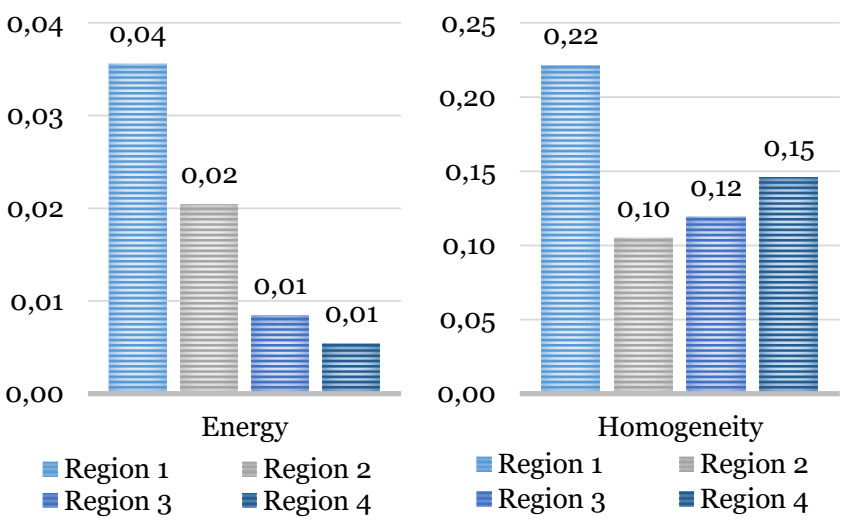

c)

d)

Fig. 10. Texture analysis: contrast (a), correlation (b), energy (c) and homogeneity (d).

The final procedure of the 2D stage found the desired ROI with certain features (see Fig. 11). As it can be seen in Fig. 11b, this step leaves only the region of the catheter and gives the output as a black and white mask which can be used for further $3 \mathrm{D}$ reconstruction.

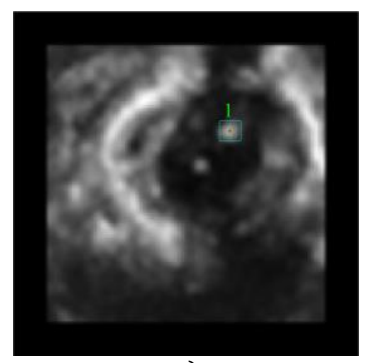

a)

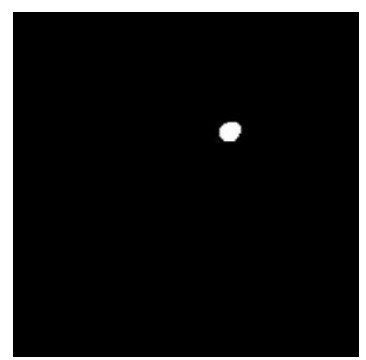

b)
Fig. 11. A detected catheter (a) and its BW mask (b).

\subsubsection{Limitations}

In order to eliminate blobs and leave the desired region of interest (ROI), we imposed general area limitations performed in the beginning: the lowest and highest area limits are 5 and 200 pixels respectively. We also performed an estimation of feature limits within one timeframe consisting of 208 slices in order to find out the distribution of lowest and highest values for further restriction. This number of slices is sufficient to figure out the feature distribution because all settings for the data acquisition were not changed within the study. In this regard, other $3 \mathrm{D}$ timeframes have relatively the same feature distribution. Feature limits 
obtained with 208 slices are shown in Fig. 12.

One of the main limitations of the algorithm is area restriction. Many excess binary large objects (blobs) frequently remain after application thresholding.

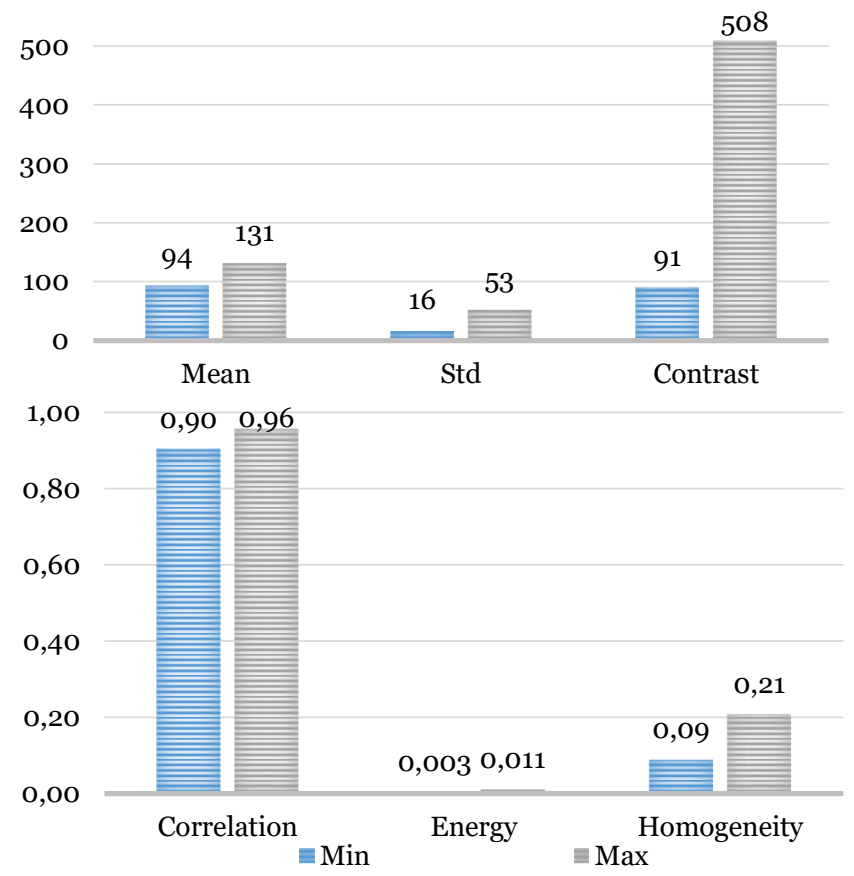

Fig. 12. Feature limits for the catheter.

\section{Results}

\subsection{Accuracy}

To assess the accuracy of the algorithm, we compared two diameters: the reference diameter and the diameter received after the application of the proposed algorithm. The catheter used for performing medical procedures in our case had a diameter equal to 7 Fr (French Gauge) or $2.333 \mathrm{~mm}$.

The diameter of the catheter was estimated using 2 ways: manual and automatic. For manual measurements, we used the short-axis view. Using this view gives a bigger sample of measurements what makes calculations of the diameter with higher precision. However, these measurements can be taken in the long-axis view but with lower accuracy. To assess the accuracy, the diameter was measured in 504 slices. To gauge the diameter automatically, we described the region of the catheter by the corresponding ellipse that has the same second moment (see Fig. 13).

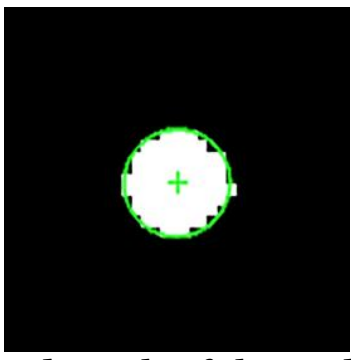

Fig. 13. Zoomed mask of the catheter region with an ellipse replacement.

As it can be seen in

Table 1, the average diameter of the catheter after executing all processing steps equals to $2.47 \mathrm{~mm}$ what is $5.84 \%$ more than the reference diameter. However, such error does not have a strong influence on the visualization because $0.14 \mathrm{~mm}$ error lets a surgeon perform medical procedures without any confusions. It was observed that Otsu's thresholding method incrassates the region of the catheter by $5-10 \%$ this is why the error is increased as well. To decrease the error, modified Otsu's method and/or more delicate threshold level should be used. It's important to note that accuracy measurement performed by the algorithm took into account position and inclination of the catheter, which allowed better orientation along the correct axes.

Table 1. Comparison of the reference catheter diameter with the diameter obtained by the proposed algorithm.

\begin{tabular}{ccc}
\hline & $\begin{array}{c}\text { Reference } \\
\text { diameter }\end{array}$ & $\begin{array}{c}\text { Diameter measured } \\
\text { by the algorithm }\end{array}$ \\
\hline Mean, mm & 2.33 & 2.47 \\
Std, mm & 0 & 0.16 \\
Error, \% & 0 & 5.84 \\
\hline
\end{tabular}

A reduction tendency of a number of regions over different steps of the proposed algorithm is shown in Table 2. According to the results, there is a trend of decreasing the number of regions. The intensity-based and area-based features reduced the number of regions approximately by half. In turn, texture feature based on the GLCM allowed excluding the false regions more accurately. Speaking of recognition accuracy and several mathematical statistics, they are shown in Table 3. The average recognition accuracy of the catheter is $87.2 \%$. The confidence level for the sample including 504 cases equals 
2.5\%. The latter means that the recognition acceptable for performing minimallyaccuracy varies from $84.6 \%$ to $89.7 \%$ with a invasive cardiac surgery. Another vital $5 \%$ significance level.

Table 2. A number of regions within different steps of the proposed algorithm.

\begin{tabular}{cc}
\hline Number of regions & Mean \pm STD \\
\hline Initial number of regions & $10.1 \pm 3.4$ \\
Number of false regions & $9.1 \pm 3.4$ \\
Number of regions after area & $8.9 \pm 3.2$ \\
restriction (low limit) & \\
Number of regions after area \\
restriction (high limit) \\
$\begin{array}{c}\text { Number of regions after } \\
\text { intensity restriction }\end{array}$ \\
$\begin{array}{c}\text { Number of regions after texture } \\
\text { restriction }\end{array}$ & $6.7 \pm 2.9$ \\
\hline
\end{tabular}

Table 3. Mathematical statistics and recognition accuracy.

\begin{tabular}{cc}
\hline Parameter & Value \\
\hline Significance level & 0.05 \\
Size & 504 \\
Confidence interval & $2.5 \%$ \\
Recognition accuracy & $87.2 \%$ \\
\hline
\end{tabular}

\subsection{Processing time}

The desktop computer with equipment described in section 2.1 was used for time assessment. We did not isolate the testing process from the influence of other processes and did not allocate a separate thread. To find the mean and standard deviation of processing time we performed 150-iteration assessment. Processing time for one timeframe is $1.96 \pm 0.045$ seconds. Each timeframe includes 208 slices what means that average processing time for $176^{*} 176$ slice is $9 \pm 0.2$ milliseconds.

\section{Conclusion}

In this research study, we developed an image-based algorithm detecting and tracking the distal end of the catheter. To correctly detect and segment the catheter, we applied a feature-based approach that can recognize the catheter along the whole $3 \mathrm{D}$ dataset. However, the algorithm works in pseudo-3D what means that it processes the data in the slice-by-slice mode. It worth noticing that the accuracy of the algorithm is at the relatively high level and equal to 94.16\%. This means that the algorithm error is 140 micrometers. However, this error is

feature of the algorithm is its processing time. Average processing time for one $3 \mathrm{D}$ timeframe is equal to 1.96 seconds and approximately 9 milliseconds per slice. Though the algorithm is not timeconsuming, it is still complicated to apply it to real-time surgery because of the huge amount of data obtained by echocardiography.

\section{Acknowledgments}

This work was supported in part by the Russian Federation Governmental Program "Nauka" № 12.8205.2017/БЧ (addition number: $\quad 4.1769$. ГЗБ.2017). The experimental calculations are carried out at Tomsk Polytechnic University within the framework of Tomsk Polytechnic University Competitiveness Enhancement Program grant.

\section{References}

1. Luani B. et al. Zero-fluoroscopy cryothermal ablation of atrioventricular nodal re-entry tachycardia guided by endovascular and endocardial catheter visualization using intracardiac echocardiography // J. Cardiovasc. Electrophysiol. 2018. Vol. 29, № 1. P. 160-166.

2. Schwein A. et al. Computed tomography angiography-fluoroscopy image fusion allows visceral vessel cannulation without angiography during fenestrated endovascular aneurysm repair // J. Vasc. Surg. 2018.

3. Cheung N.K. et al. Radiation exposure, and procedure and fluoroscopy times in endovascular treatment of intracranial aneurysms: a methodological comparison // J. Neurointerv. Surg. 2018. P. neurintsurg-2017-013596.

4. Spitzer E. et al. The Role of Automated 3D Echocardiography for Left Ventricular Ejection Fraction Assessment // Card. Fail. Rev. 2017. Vol. 3, № 2. P. 97.

5. Muraru D. et al. Three-dimensional 
speckle-tracking echocardiography: benefits and limitations of integrating myocardial mechanics with threedimensional imaging // Cardiovasc. Diagn. Ther. 2018. Vol. 8, № 1. P. 101117.

6. Tepeler A. et al. Factors affecting the fluoroscopic screening time during percutaneous nephrolithotomy // J Endourol. 2009. Vol. 23, № 11. P. 1825-1829.

7. Hoffmann M. et al. Semi-automatic catheter reconstruction from two views. // Med. image Comput. Comput. Interv. 2012. Vol. 15, № Pt 2. P. 584-591.

8. Hoffmann M. et al. Electrophysiology catheter detection and reconstruction from two views in fluoroscopic images // IEEE Trans. Med. Imaging. 2016. Vol. 35, № 2. P. 567-579.

9. Baert S.A.M. et al. Three-Dimensional Guide-Wire Reconstruction from Biplane Image Sequences for Integrated Display in 3-D Vasculature // IEEE Trans. Med. Imaging. 2003. Vol. 22, № 10. P. 1252-1258.

10. Ma Y. et al. Real-time x-ray fluoroscopy-based catheter detection and tracking for cardiac electrophysiology interventions. // Med. Phys. 2013. Vol. 40, № 7. P. 071902.

11. Ambrosini P. et al. A Hidden Markov Model for $3 \mathrm{D}$ Catheter Tip Tracking with 2D X-ray Catheterization Sequence and 3D Rotational Angiography // IEEE Trans. Med. Imaging. 2017. Vol. 36, № 3. P. 757768.

12. Wu X. et al. Catheter tracking in $3 \mathrm{D}$ echocardiographic sequences based on tracking in 2D X-ray sequences for cardiac catheterization interventions // Proceedings - International Symposium on Biomedical Imaging. 2013.

13. Otsu N. A Threshold Selection Method from Gray-Level Histograms // IEEE Trans. Syst. Man. Cybern. 1979. Vol. 9, № 1. P. 62-66.

14. Soille P. Morphological Image
Analysis. Berlin, Heidelberg: Springer Berlin Heidelberg, 2004. 392 p.

15. Haralick R.M., Shapiro L.G. Computer and Robot Vision // Computer and Robot Vision. 1992. Vol. 1. 28-48 p.

16. Haralick R.M., Shanmugam K., Dinstein I. Textural Features for Image Classification // IEEE Trans. Syst. Man. Cybern. 1973. Vol. SMC-3, № 6. P. 610-621.

17. Rampun A., Strange H., Zwiggelaar R. Texture segmentation using different orientations of GLCM features // Proceedings of the 6th International Conference on Computer Vision / Computer Graphics Collaboration Techniques and Applications MIRAGE '13. 2013.

18. Nagarajan M.B. et al. Classification of small lesions in dynamic breast MRI: eliminating the need for precise lesion segmentation through spatio-temporal analysis of contrast enhancement // Mach. Vis. Appl. 2013. Vol. 24, № 7. P. 1371-1381. 